\title{
The functional consequences of age-related changes in microRNA expression in skeletal muscle
}

\author{
Ana Soriano-Arroquia $\cdot$ Louise House $\cdot$ Luke Tregilgas • \\ Elizabeth Canty-Laird · Katarzyna Goljanek-Whysall (i)
}

Received: 28 July 2015/Accepted: 18 February 2016/Published online: 27 February 2016

(C) The Author(s) 2016. This article is published with open access at Springerlink.com

\begin{abstract}
A common characteristic of ageing is disrupted homeostasis between growth and atrophy of skeletal muscle resulting in loss of muscle mass and function, which is associated with sarcopenia. Sarcopenia is related to impaired balance, increased falls and decline in quality of life of older people. Ageingrelated transcriptome and proteome changes in skeletal muscle have been characterised, however the molecular mechanisms underlying sarcopenia are still not fully understood. microRNAs are novel regulators of gene expression known to modulate skeletal muscle development and homeostasis. Expression of numerous microRNAs is disrupted in skeletal muscle with age however, the functional consequences of this are not yet understood. Given that a single microRNA can simultaneously affect multiple signalling pathways, microRNAs are potent modulators of
\end{abstract}

Ana Soriano-Arroquia and Louise House these authors contributed equally to this work.

Electronic supplementary material The online version of this article (doi:10.1007/s10522-016-9638-8) contains supplementary material, which is available to authorized users.

A. Soriano-Arroquia · L. House - L. Tregilgas ·

E. Canty-Laird · K. Goljanek-Whysall ( $\square)$

MRC-Arthritis Research UK Centre for Integrated

research into Musculoskeletal Ageing (CIMA),

Department of Musculoskeletal Biology, Institute of

Ageing and Chronic Disease, University of Liverpool,

Apex Building, West Derby Road, Liverpool L8 7TX, UK

e-mail: k.whysall@liverpool.ac.uk pathophysiological changes occurring during ageing. Here we use microRNA and transcript expression profiling together with microRNA functional assays to show that disrupted microRNA:target interactions play an important role in maintaining muscle homeostasis. We identified miR-181a as a regulator of the sirtuin1 (Sirt1) gene expression in skeletal muscle and show that the expression of miR-181a and its target gene is disrupted in skeletal muscle from old mice. Moreover, we show that miR-181a:Sirt1 interactions regulate myotube size. Our results demonstrate that disrupted microRNA:target interactions are likely related to the pathophysiological changes occurring in skeletal muscle during ageing.

Keywords microRNA $\cdot$ Sarcopenia $\cdot$ Sirtl $\cdot$ Muscle . Ageing

\section{Introduction}

Age-related loss of skeletal muscle mass and function, associated with sarcopenia, results in frailty, decline in strength and decrease in quality of life of older people. The mechanisms of age-related defective muscle homeostasis related to hypertrophy/atrophy are multifactorial and depend on a number of changes including mitochondrial production of reactive oxygen species (Jackson and McArdle 2011), changes in the muscle niche (Carlson and Conboy 2007), or alterations in circulating factors (Carlson et al. 2009). 
Moreover, skeletal muscles of adult and old animals display substantially different gene (Welle et al. 2003; Sifakis et al. 2013) and protein (McDonagh et al. 2014) expression profiles.

microRNAs (miRNAs) are small, non-coding RNAs that regulate gene expression at the posttranscriptional level. miRNAs are predicted to regulate two-thirds of the human genome, suggesting that miRNAs modulate many physiologically relevant processes (Friedman et al. 2009). Mature miRNAs are generated from primary-miRNA (pri-miRNA) precursors which are cleaved by the enzyme Drosha forming the pre-miRNA transcript. The pre-miRNA is transported into the cytoplasm, where the enzyme Dicer generates a 19-24 base pair miRNA duplex (Bartel 2004). The mature miRNA strand is incorporated into the RISC complex (RNA Induced Silencing Complex). The non-incorporated strand is often degraded however it may also be incorporated into the RISC. miRNAs guide the RISC to partially complementary sequences, usually contained within the $3^{\prime}$ UTR of target mRNA transcripts, resulting in target mRNA degradation and/or inhibition of translation. Most mammalian miRNAs have only partially complementary sequences to their target mRNAs (Bartel 2004) resulting in challenging bioinformatic prediction of the target genes.

microRNAs play significant roles in myogenic processes during embryonic development (GoljanekWhysall et al. 2011, 2014) and in adults by regulating satellite cell function, muscle hypertrophy and myofibre type (McCarthy and Esser 2007; Crist et al. 2009; Van Rooij et al. 2009b; Goljanek-Whysall et al. 2012; Brown and Goljanek-Whysall 2015; Soriano-Arroquia et al. 2016). Moreover, the role of miRNAs in muscle during ageing has been demonstrated using satellite cell specific Dicer-deficient mice (Cheung et al. 2012), characterised by mild muscle atrophy and an impaired ability to regenerate muscle fibres following muscle injury. The differential expression of many muscle-enriched as well as non-muscle specific miRNAs in skeletal muscle during ageing in multiple species, including mice (Kim et al. 2014a), rats (Hu et al. 2014a), rhesus monkeys (Mercken et al. 2013) and humans (Drummond et al. 2011a; Rivas et al. 2014b; Zacharewicz et al. 2014) has been demonstrated. Moreover, differential expression of miRNAs in skeletal muscle of adult and old humans in response to an acute bout of resistance exercise has been shown (Rivas et al. 2014a; Zacharewicz et al. 2014). Soares et al. (2014) recently demonstrated that different catabolic conditions in muscle are associated with a unique and dynamic miRNA signature (Soares et al. 2014).

In this study, we aimed to establish the potential functional consequences of age-related dysregulation of microRNA:target interactions in skeletal muscle during ageing. We characterised the global changes in microRNA and mRNA expression profiles in skeletal muscle of adult and old mice using bioinformatics tools and modelling. Moreover, we identified a group of microRNAs, expression of which is affected by ageing in mice and humans and validated the ageingassociated changes in their expression in mouse skeletal muscle. We demonstrated that miR-181a is predicted to play a central role in ageing-related disrupted muscle homeostasis and validated differential expression of microRNA-181a and its target genes in skeletal muscle during ageing. Finally, we validated Sirtl as a miR-181a target and demonstrated that manipulation of miR-181a expression regulates myotube size in vitro. These data suggest that ageingassociated changes in miR-181a and expression of its target gene(s) may indeed be associated with the ageing-related disrupted balance between muscle hypertrophy and atrophy.

\section{Materials and methods}

Mice

The study was performed using male wild type C57Bl/ 6 mice obtained from Charles River (Margate). All mice were maintained under specific-pathogen free conditions, fed ad libitum a standard chow and maintained under barrier on a 12-h light-dark cycle. For tissue collection, mice were culled by cervical dislocation. The tissues were immediately excised, frozen and stored at $-80{ }^{\circ} \mathrm{C}$. Experiments were performed in accordance with UK Home Office guidelines under the UK Animals (Scientific Procedures) Act 1986 and received ethical approval from the University of Liverpool Animal Welfare and Ethical Review Body (AWERB). For microarray studies, $\mathrm{n}=3$ adult ( 6 month) and $\mathrm{n}=3$ old ( 24 month) mice were used; for qPCR validation, an independent cohort of $\mathrm{n}=6-9$ adult and $\mathrm{n}=4-6$ old mice was used. 
microRNA and mRNA expression profiling in skeletal muscle of adult and old mice

The tibialis anterior muscles (30-40 mg each) from adult and old mice were dissected and RNA was isolated using Trizol as described below. RNA integrity was assessed using a Bioanalyser instrument (Agilent Technologies) and samples with RIN $>7$ were used. An Affymetrix FlashTag Labelling kit and GeneChip microRNA 3.0 arrays were used for global miRNA expression profiling. GeneChip Mouse Gene 2.0 ST arrays (Affymetrix) were used for global transcriptomic analysis. Data pre-processing, modelling and differential expression analyses were carried out using the Expression Console and Transcriptome Analysis Control (Affymetrix) in the $\mathrm{R}$ environment (http://cran.r-project.org) using packages from the Bioconductor project (http://www. bioconductor.org). The oligo package (Carvalho and Irizarry 2010) has been developed for use with Affymetrix Gene ST arrays and takes into account the lack of incorporation of mismatch probes into the chip architecture. Raw Data distributions were initially visualised and assessed for spatial artefacts, array clustering, technical variability between arrays and to identify outliers prior to carrying out background correction, normalisation and summarisation using Robust Multichip Averaging (RMA). There were no outlying samples identified in the dataset, therefore $\mathrm{n}=3$ persists through to the differential expression analysis and the full sample set is represented. Data was then filtered for low variance using the genefilter package. Modelling and statistical analysis of differentially expressed genes was carried out in the limma package (Smyth 2004; Smyth et al. 2005; Ritchie et al. 2006, 2015). Two-group comparisons were used to determine statistically significant, differentially expressed transcript clusters using an empirical Bayes moderated $t$ test. The Benjamini-Hochberg method was used for false discovery rate correction of p-values. For changes in microRNA expression in human skeletal muscle, publically available datasets were used (GSE23527; (Drummond et al. 2011b; Rivas et al. 2014a).

microRNA:target interaction predictions

Analysis of differentially expressed microRNAs and mRNAs and predicted microRNA target genes was completed using QIAGEN's Ingenuity ${ }^{\circledR}$ Pathway Analysis $\left(\mathrm{IPA}^{\circledR}\right.$ ) Product (Ingenuity ${ }^{\circledR}$ Systems, http://www.ingenuity.com), specifically the Core Analysis function, the Path Designer feature and the microRNA Target Filter. A list of experimentally validated and predicted microRNA targets was produced based on Targetscan Human, TarBase, miRecords, Ingenuity ${ }^{\circledR}$ Expert Findings and Ingenuity ${ }^{\circledR}$ ExpertAssist Findings.

\section{Cell culture and transfections}

C2C12 cells were cultured in Dulbecco's modified medium (DMEM) supplemented with $10 \%$ fetal bovine serum (FBS), $1 \%$ L-glutamine and $1 \%$ penicillin/streptomycin. To induce myogenic differentiation, $90 \%$ confluent cells were cultured in DMEM supplemented with $2 \%$ horse serum, $1 \%$ L-glutamine and $1 \%$ penicillin/streptomycin (Goljanek-Whysall et al. 2012).

Myotubes were transfected with either $100 \mathrm{nM}$ miRNA-181, $100 \mathrm{nM}$ antimiR-181 or $2.5 \mu \mathrm{g}$ SIRT1 overexpression vector (Addgene, 1791) (Brunet et al. 2004) using Lipofectamine $2000^{\mathrm{TM}}$. Mock-transfected or antimiR-scrambled-transfected cells served as controls. Transfection efficiency was 40-50\%, depending on the molecule transfected as described previously (Goljanek-Whysall et al. 2012). To assess the changes in myotube diameter following the transfections, MF20 (myosin heavy chain) immunostaining was performed at 4 days post- transfection.

\section{Real-Time PCR and western blotting}

RNA isolation and quantitative real time PCR were performed using standard methods as described previously (Goljanek-Whysall et al. 2014). cDNA synthesis (mRNA) was performed using 400-500 ng RNA and SuperScript II according to the manufacturer's protocol. cDNA synthesis (microRNA) was performed using 100 ng RNA and miRscript RT kit II according to the manufacturer's protocol. microRNA qPCR analysis was performed using miRScript SybrGreen Mastermix in a $20 \mu \mathrm{l}$ reaction according to the manufacturer's protocol. mRNA qPCR was performed using sso-Advanced SybrGreen Mastermix, using $1 \mu \mathrm{l}$ of $10 \mu \mathrm{M}$ each primer in $20 \mu \mathrm{l}$ reaction. All amplicons were designed to span an intron-exon junction (where mRNAs consisted of more than one exon) and were 
200-300 nt long. Expression relative to $\beta-2$ microglobulin and/or $18 S$ (mRNA) or Rnu-6 and/or Snord-61 (microRNA) was calculated using the delta delta $\mathrm{Ct}$ method. The qPCR conditions were: $95^{\circ} \mathrm{C}$ $10 \mathrm{~s}, 55^{\circ} \mathrm{C}$ (miRNA) or $60{ }^{\circ} \mathrm{C}$ (mRNA) $30 \mathrm{~s}, 72{ }^{\circ} \mathrm{C}$ $30 \mathrm{~s}$ (40x). Protein lysis and Western blots were performed as described (Goljanek-Whysall et al. 2014). The primers and reagents used are listed in Tables S4 and S5, respectively.

In vitro miRNA target validation

Predicted target genes of miR-181 were initially chosen based on the global profiling data. Next our own and published data were used to narrow down the list of potential candidate targets to the ones that may be relevant to muscle ageing. The $3^{\prime}$ UTR region of Sirt 1 with a wild type (WT) or mutated miR-181 target site (mutant) were synthesised using GeneArt service (Invitrogen) and cloned into a GFP TOPO vector (Invitrogen). $\mathrm{C} 2 \mathrm{C} 12$ myotubes cultured in 6-well plates were transfected using Lipofectamine $2000^{\mathrm{TM}}$ with WT or mutant constructs (1000 ng), with or without miRNA mimic $(50 \mathrm{nM})$. Protein was extracted after $48 \mathrm{~h}$ and GFP expression analysed by Western blotting.

\section{Results}

Ageing affects microRNA:target interactions in skeletal muscle of mice

To model microRNA:target interactions in skeletal muscle during ageing, an unbiased microRNA and mRNA expression profiling was performed on the tibilais anterior (TA) muscle of adult and old mice using microarrays (Figure S1). The expression of 16 microRNAs was significantly downregulated and the expression of 14 microRNAs was significantly upregulated in muscle during ageing (Table 1). Among the differentially expressed microRNAs were miR-181a, miR-208-5p or miR-499, miR-130a, miR-26a and miR-30c with previously characterised functions in skeletal muscle.

Given the potential of miRNAs to regulate a large number of cellular transcripts, transcript expression profiling was performed. The expression of only 12 transcripts was significantly downregulated and the expression of 19 mRNAs was significantly upregulated in the TA muscle of old mice compared with the muscle of adult mice $(\mathrm{p}<0.05$; Table 2$)$. This low number of differentially expressed genes in muscle during ageing may be related to a limited number of
Table 1 microRNAs differentially expressed in the tibialis anterior muscle of mice during ageing
microRNA expression was assessed in the TA muscle of adult and old mice; the microRNA identity and fold change are given.

MicroRNAs previously noted to be differentially expressed in human skeletal muscle during ageing are highlighted in red; adult: 6 months old; old:

24 months old; $\mathrm{n}=3$

\begin{tabular}{lcllll}
\hline $\begin{array}{l}\text { microRNA } \\
\text { downregulated } \\
\text { during ageing }\end{array}$ & $\begin{array}{l}\text { Fold Change } \\
\text { (Old vs Adult) }\end{array}$ & $\mathrm{p}$ value & $\begin{array}{l}\text { microRNA } \\
\text { upregulated during } \\
\text { ageing }\end{array}$ & $\begin{array}{l}\text { Fold Change } \\
\text { (Old vs Adult) }\end{array}$ & $\mathrm{p}$ value \\
\hline miR-122 & -19.64 & 0.039 & miR-26a & 1.49 & 0.0004 \\
miR-382 & -6.17 & 0.038 & mir-499 & 1.48 & 0.001 \\
miR-148a & -4.31 & 0.038 & miR-5103 & 1.32 & 0.002 \\
miR-344f-5p & -3.41 & 0.038 & miR-34b-3p & 3.15 & 0.002 \\
miR-132 & -2.63 & 0.003 & miR-669 g & 1.31 & 0.003 \\
miR-379 & -2.39 & 0.028 & mir-669o & 1.76 & 0.004 \\
miR-301a & -2.26 & 0.044 & miR-217* & 1.12 & 0.005 \\
miR-30c* & -2.06 & 0.00002 & mir-5123 & 1.63 & 0.005 \\
miR-127 & -2.05 & 0.004 & miR-1186 & 2.05 & 0.008 \\
mir-181a & -1.52 & 0.001 & miR-431* & 2.51 & 0.009 \\
miR-208a-5p & -1.5 & 0.00 & mir-669 m & 2.15 & 0.016 \\
mir-3074-1 & -1.36 & 0.005 & miR-467b* & 3.1 & 0.016 \\
miR-471-3p & -1.32 & 0.002 & miR-133a* & 2.39 & 0.033 \\
miR-3475 & -1.29 & 0.004 & miR-872* & 2.23 & 0.049 \\
miR-465c-3p & -1.19 & 0.0006 & & & \\
mir-3063 & -1.18 & 0.004 & & & \\
\hline
\end{tabular}


Table 2 Transcripts differentially expressed in the tibialis anterior muscle of mice during ageing

\begin{tabular}{|c|c|c|c|}
\hline $\begin{array}{l}\text { Gene Symbol (transcripts } \\
\text { downregulated) }\end{array}$ & $\begin{array}{l}\text { Fold change } \\
\text { (Old vs Adult) }\end{array}$ & $\mathrm{p}$ value & Description \\
\hline Krt10 & -7.61 & 0.030813 & keratin 10 \\
\hline Arrdc2 & -4.87 & 0.034934 & arrestin domain containing 2 \\
\hline Dsc1 & -3.55 & 0.033618 & desmocollin 1 \\
\hline Dsg1a & -3.51 & 0.042671 & desmoglein 1 alpha \\
\hline Krt77 & -3.31 & 0.046219 & keratin 77 \\
\hline Hrnr & -3.07 & 0.014021 & hornerin \\
\hline Gm24202 & -3.05 & 0.04403 & predicted gene, 24202 \\
\hline Pof1b & -2.93 & 0.039252 & premature ovarian failure $1 \mathrm{~B}$ \\
\hline Mettl21c & -2.88 & 0.008667 & methyltransferase like $21 \mathrm{C}$ \\
\hline Ucp3 & -2.75 & 0.003941 & uncoupling protein 3 (mitochondrial, proton carrier) \\
\hline Neto2 & -2.61 & 0.046314 & neuropilin (NRP) and tolloid (TLL)-like 2 \\
\hline $\mathrm{Gm} 24128$ & -2.4 & 0.032397 & predicted gene, 24128 \\
\hline Lce1 m & -2.39 & 0.014247 & late cornified envelope $1 \mathrm{M}$ \\
\hline Slc25a30 & -2.37 & 0.006328 & solute carrier family 25 , member 30 \\
\hline Gadd45g & -2.34 & 0.001227 & growth arrest and DNA-damage-inducible 45 gamma \\
\hline Nrep & -2.1 & 0.003032 & neuronal regeneration related protein \\
\hline Rarres 1 & -2.04 & 0.037197 & retinoic acid receptor responder (tazarotene induced) 1 \\
\hline Zfp640 & -2.02 & 0.03305 & zinc finger protein 845-like; zinc finger protein 640 \\
\hline Pdk4 & -2.01 & 0.002642 & pyruvate dehydrogenase kinase, isoenzyme 4 \\
\hline $\begin{array}{l}\text { Gene Symbol } \\
\text { (transcripts upregulated) }\end{array}$ & $\begin{array}{l}\text { Fold Change } \\
\text { (Old vs Adult) }\end{array}$ & p-value & Description \\
\hline Krt18 & 3.76 & 0.002333 & keratin 18 \\
\hline Cpne2 & 3.02 & 0.002247 & copine II \\
\hline Igkv4-57-1 & 2.75 & 0.023636 & immunoglobulin kappa variable 4-57-1 \\
\hline Nabp1 & 2.49 & 0.007488 & nucleic acid binding protein 1 \\
\hline Cilp & 2.48 & 0.002913 & $\begin{array}{l}\text { cartilage intermediate layer protein, nucleotide } \\
\text { pyrophosphohydrolase }\end{array}$ \\
\hline Tnfrsf 23 & 2.41 & 0.005439 & tumor necrosis factor receptor superfamily, member 23 \\
\hline Rrad & 2.25 & 0.009309 & Ras-related associated with diabetes \\
\hline Cdkn1a & 2.21 & 0.018982 & cyclin-dependent kinase inhibitor 1A (P21) \\
\hline Gm6821 & 2.19 & 0.009309 & predicted gene 6821 \\
\hline Ankrd1 & 2.17 & 0.008222 & ankyrin repeat domain 1 (cardiac muscle) \\
\hline Gm26179 & 2.11 & 0.035369 & predicted gene, 26179 \\
\hline Trim30a & 2.1 & 0.006883 & tripartite motif-containing 30A \\
\hline
\end{tabular}

Target name and description, fold change in expression and $\mathrm{p}$ value are given; adult 6 months old; old 24 months old; $\mathrm{n}=3$

biological replicates $(n=3)$. Among the genes differentially expressed during ageing were genes associated with metabolism and mitochondrial function: Ucp3, Pdk4; methylation: Mettl21c; DNA damage response: Gadd45 g; retinoic acid signalling: Rarres 1 ; as well as cellular senescence: $C d k n 1(\mathrm{p} 21)$.
To understand the biological relevance of the changes in microRNA expression during ageing, microRNA: predicted target interactions were modelled using miRSystem (http://mirsystem.cgm.ntu. edu.tw/index.php). The top pathway predicted to be regulated by microRNAs downregulated in muscle 
during ageing was the insulin signalling pathway with 137 microRNA putative targets being associated with this pathway (Table 3). Targets of microRNAs upregulated during ageing were associated with protein processing in endoplasmic reticulum, splicing and ubiquitin-mediated proteolysis (Table 3).

Analysis of microRNA:target interactions using Ingenuity pathways indicated that ubiquitin-mediated proteolysis, mitochondrial metabolism, insulin signalling and splicing were regulated by microRNAs downregulated in muscle during ageing, whereas cell senescence, TGF $\beta$ (transforming growth factor beta), NF-kB (nuclear factor $\kappa B$ ) and MAPK (mitogenactivated protein kinase) signalling were mainly regulated by microRNAs upregulated in muscle during ageing (Fig. 1). Further pathway analysis using Ingenuity was performed to determine which miRNAs and their target genes form networks of connections. We observed that there were several genes and microRNAs forming nodes in the interaction network that comprised differentially expressed mRNAs and microRNAs and their predicted target genes (Fig. S2). Genes associated with ubiquitin-mediated proteolysis, cell cycle, NF-kB and insulin signalling, as well as miRs miR-132, miR-122, miR-499, miR-128 and miR-22 formed central nodes in the network of miRNA:target interactions disrupted during ageing (Fig. S2).

Overall, these data indicate that several miRNA:target interactions are deregulated in skeletal muscle during ageing. These changes are likely to contribute
Fig. 1 Disrupted microRNA:target interaction networks in skeletal muscle of mice during ageing. The network of interactions was constructed using IPA software. The inputs were microRNAs and their predicted targets and mRNAs differentially expressed in muscle during ageing. In red miRs/genes that were downregulated with age are shown; in green miRs/genes that were upregulated with age are shown. a. Networks of interactions associated with miRs downregulated in muscle during ageing. b. Networks of interactions associated with miRs upregulated in muscle during ageing

to ageing-related pathophysiological processes, such as loss of muscle mass and function through regulation of ubiquitin-mediated proteolysis, ER stress and NF$\mathrm{\kappa b}$ signalling, senescence associated with disrupted cell cycle or myofibre hypertrophy associated with insulin signalling.

A set of microRNAs is deregulated during ageing in muscle of human and mice

Publically available datasets were examined for differential expression of microRNAs in human skeletal muscle during ageing (Drummond et al. 2011b; Rivas et al. 2014a) in order to establish whether a specific set of miRNAs was affected by ageing in both mouse and human skeletal muscle. Only 7 microRNAs were consistently deregulated during ageing in human and mouse skeletal muscle (Table 1; miRs highlighted in red). Ingenuity pathways analysis indicated that differential expression of these 7 microRNAs and consequently their target genes may result in defective insulin, MAPK and TGF $\beta$
Table 3 Functional annotation of predicted targets of microRNAs differentially expressed in skeletal muscle of mice during ageing; termfunctional annotation term associated with miRNA predicted target genes

\begin{tabular}{lcc}
\hline TERM (Predicted targets of downregulated mirnas) & Total genes & p value \\
\hline Insulin signaling pathway & 137 & $4.05 \mathrm{E}-02$ \\
Carbohydrate digestion and absorption & 39 & $4.06 \mathrm{E}-02$ \\
Starch and sucrose metabolism & 44 & $4.45 \mathrm{E}-02$ \\
Type ii diabetes mellitus & 49 & $4.95 \mathrm{E}-02$ \\
Amino sugar and nucleotide sugar metabolism & 47 & $6.56 \mathrm{E}-02$ \\
Glycolysis gluconeogenesis & 60 & $9.10 \mathrm{E}-02$ \\
Fructose and mannose metabolism & 36 & $9.26 \mathrm{E}-02$ \\
Galactose metabolism & 27 & $2.62 \mathrm{E}-01$ \\
TERM (Predicted targets of upregulated mirnas) & & \\
Endoplasmic reticulum & 167 & $3.01 \mathrm{E}-01$ \\
Rna transport & 150 & $2.82 \mathrm{E}-01$ \\
Ubiquitin mediated proteolysis & 138 & $1.59 \mathrm{E}-01$ \\
Spliceosome & 126 & $3.25 \mathrm{E}-01$ \\
Erbb signaling pathway & 87 & $6.63 \mathrm{E}-02$ \\
\hline
\end{tabular}




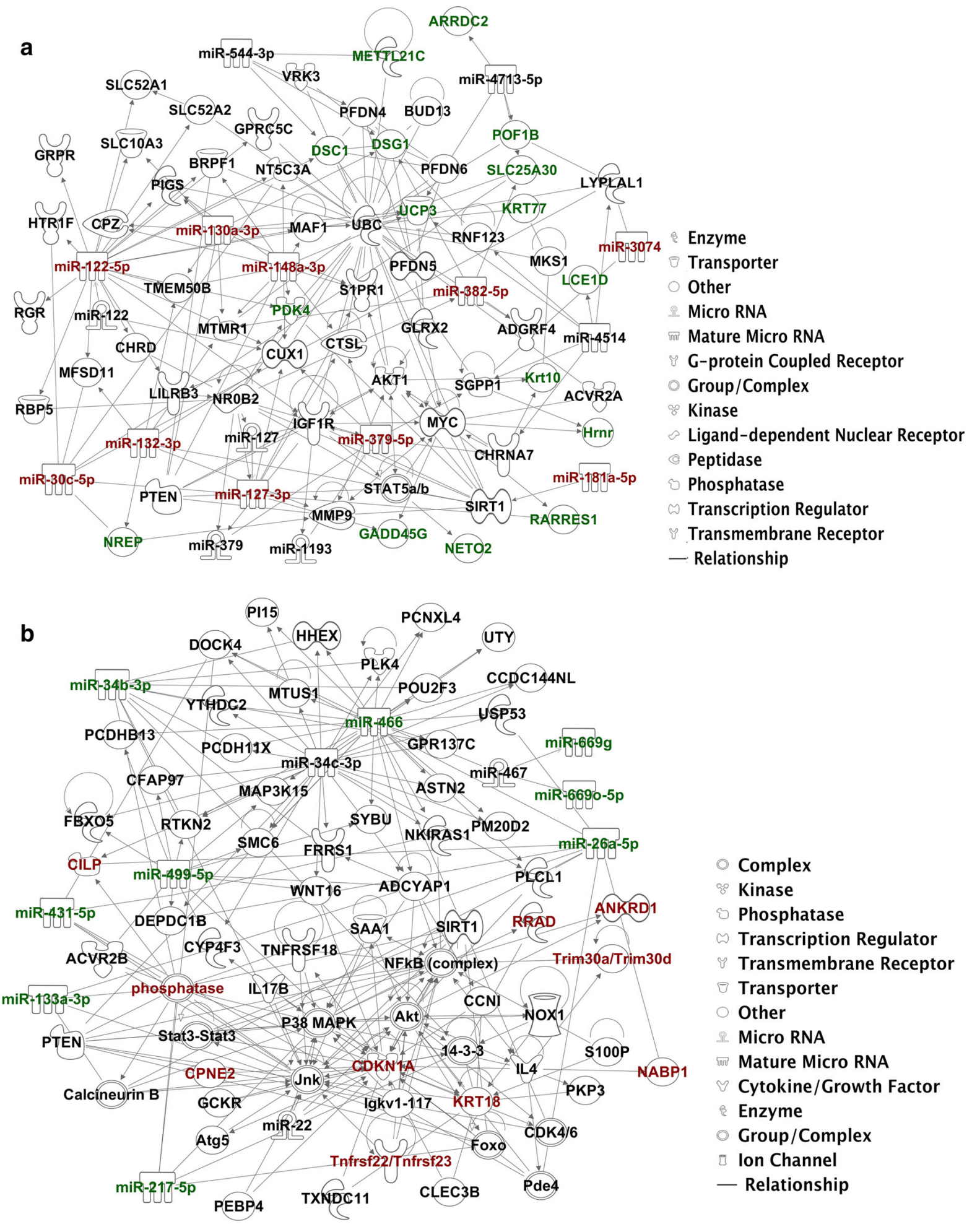


Fig. 2 Disrupted microRNA:target interaction network in skeletal muscle of humans and mice during ageing. The network of interactions was constructed using IPA software. The input was microRNAs and their experimentally validated targets

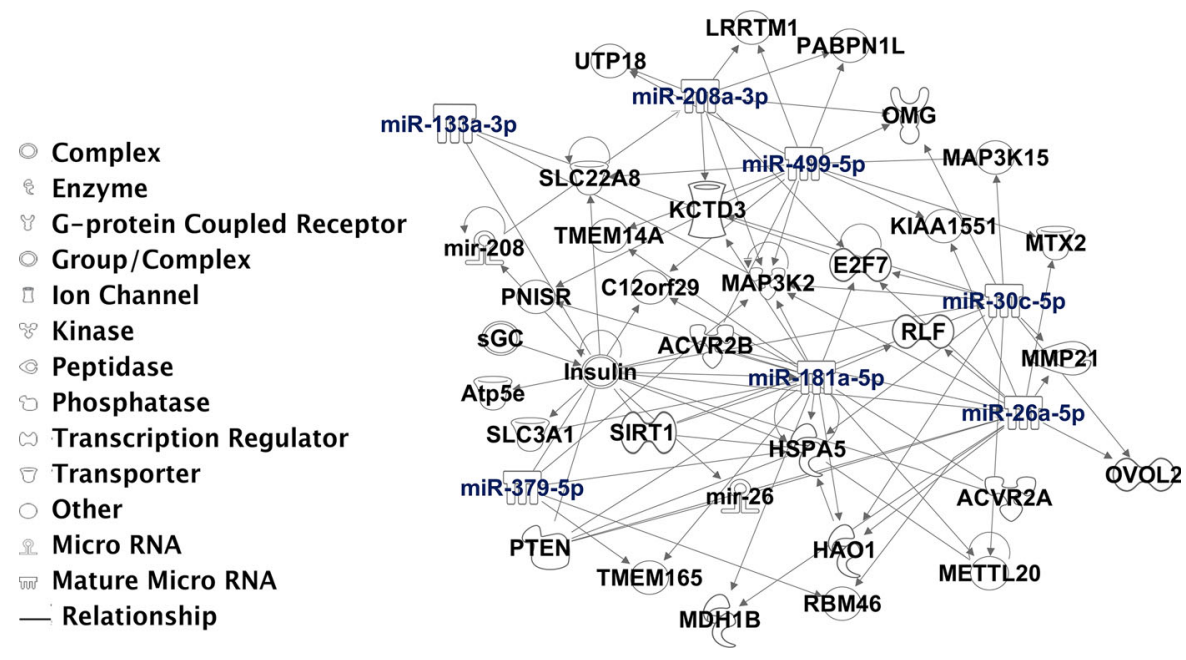

To establish whether miR-181 directly interacts with the Sirt1 3'UTR, we generated a reporter construct containing a fragment of the Sirt1 3'UTR downstream of a GFP reporter ("wild type"). "Mutant" reporter contained a mutated miR-181 binding site. The GFP reporter containing wild type Sirtl 3'UTR was efficiently regulated by miR-181 but not by miR-24; a microRNA not predicted to target Sirt1 (negative control) (Fig. 4b, c). Mutation of the putative target site in the $3^{\prime} \mathrm{UTR}$ rendered the reporter construct insensitive to miR-181, indicating that interaction with the target site is required for the response (Fig. 4b, c).

To validate Sirt 1 as a physiologically relevant miR181 target gene in muscle, the expression of Sirt1 transcript and protein was examined in $\mathrm{C} 2 \mathrm{C} 12$ myotubes following miR-181 overexpression or inhibition using miRNA mimic or antimiR (AM181), respectively (Fig. 4c, d). The efficiency of the transfections was validated (Fig. S4). The expression of SIRT1 protein, but not mRNA in $\mathrm{C} 2 \mathrm{C} 12$ myotubes was downregulated following overexpression of miR-181 and upregulated following inhibition of miR-181 function (Fig. 4d-f). These data show that miR-181a directly regulated SIRT1 expression at the protein level.

Changes in miR-181a:Sirt1 affect myotube size

To establish whether age-related changes in miR-181a expression may have functional consequences on muscle homeostasis, $\mathrm{C} 2 \mathrm{C} 12$ myotubes were used as an in vitro model to study myotube hypertrophy and/or atrophy. C2C12 myotubes were transfected with 
a
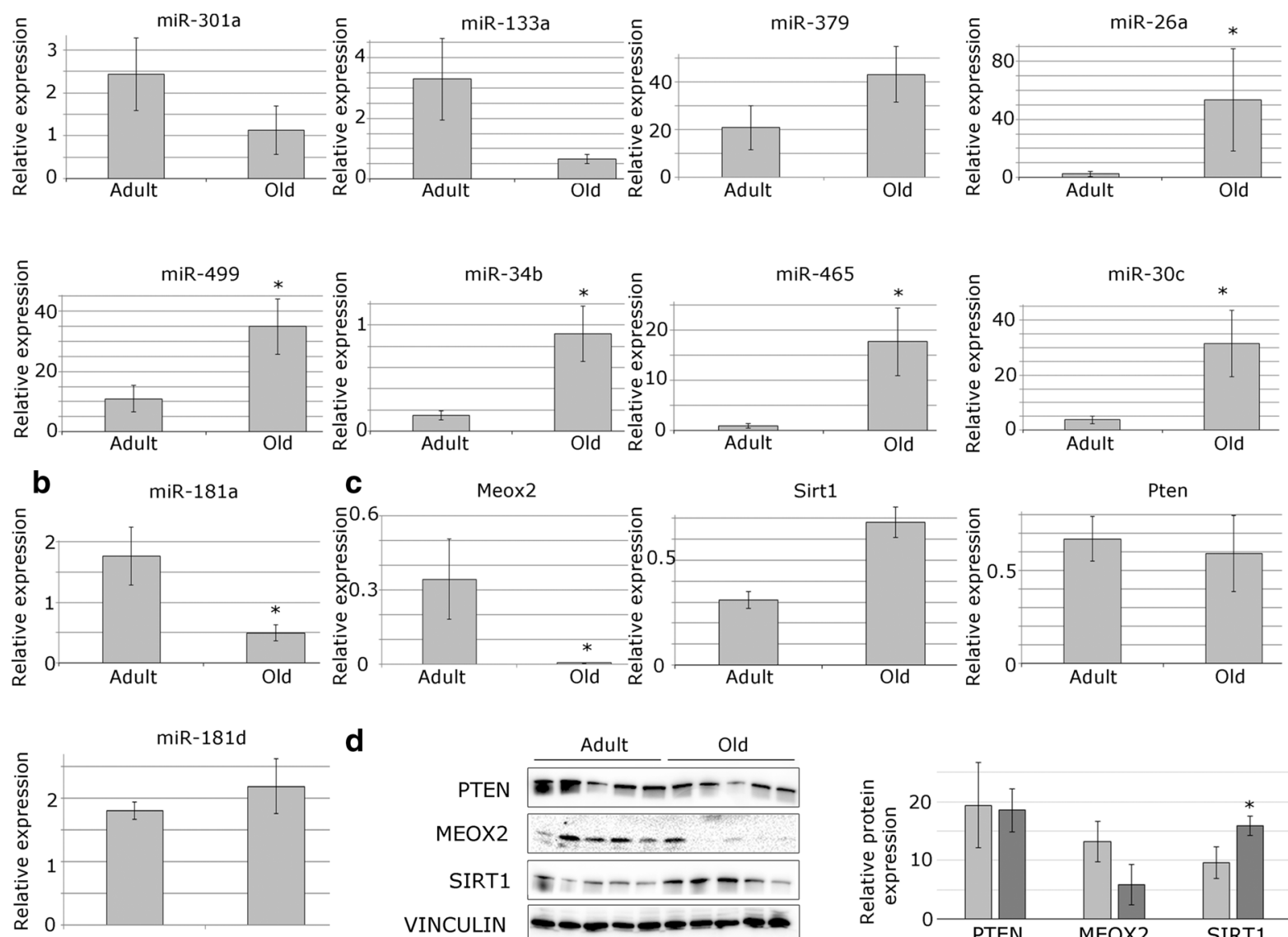

d
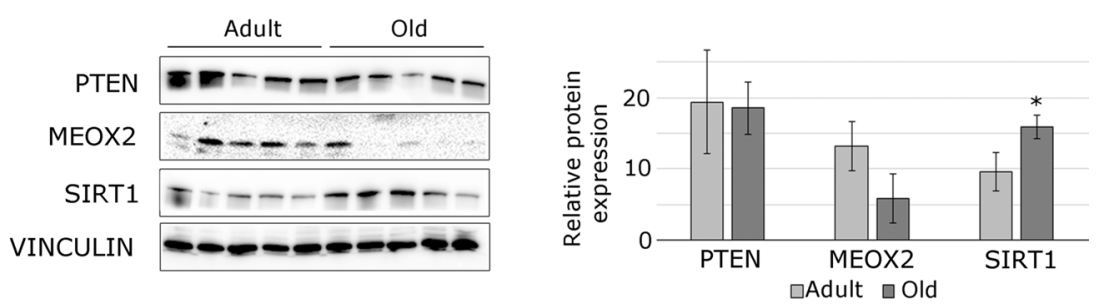

Fig. 3 Validation of the differential expression of miRNAs and target mRNAs in muscle during ageing. a qPCR showing expression (relative to Rnu-6) of miRs differentially expressed in skeletal muscle of mice and/or humans. b qPCR showing relative expression of miR-181a and miR-181d relative to Rnu-6 in TA muscle of mice during ageing. $\mathbf{c}$ qPCR showing relative

miR-181 mimic or inhibitor (AM) or SIRT1 overexpression construct. At 4 days following transfection myotubes were stained for myosin heavy chain (MF20) and myotube diameter measured. miR-181a overexpression led to a significant decrease in myotube diameter, whereas miR-181a inhibition led to an increase in myotube diameter as compared to mock- and scrambled-transfected controls (Fig. 5). SIRT1 overexpression resulted in an increased myotube diameter as compared to mock- and scrambled- transfected controls (Fig. 5). Co-transfection of SIRT1 overexpression construct together with miR181 mimic rescued the miR-181-induced phenotype, (to $\beta$-2-microglobulin) expression of miR-181a predicted target genes in muscle of mice during ageing. d Western blot and quantification showing differential protein expression of miR181a predicted targets in TA muscle of mice during ageing. Representative Western blots are shown. Error bars show SEM; $\mathrm{n}=4-7 ; * \mathrm{p}<0.05$

indicating the importance of Sirtl as miR-181 target gene in controlling myotube size (Fig. 5).

The effect of changes in miR-181 and SIRT1 expression on the expression of $p 21$, a cell cycle regulator associated with senescence and upregulated in muscle of older mice, was examined (Table 2; Fig. S5). We did not detect significant changes in $p 21$ mRNA expression following manipulation of miR181a levels, however $p 21$ expression was downregulated following SIRT1 upregulation in C2C12 myotubes indicating that SIRT1 may play additional, miR181-independent function in muscle, such as regulating cell senescence (Fig. S5). 


\section{a 3'UGAGUGGCUGUCGCAACUUACA Д' mmu-miR-181a \\ 5' UUAGCAUGUAAAAAAUGAAUGU3' mmu-Sirt1 \\ 5'UUUAGCAUGUCAAAAUGAAUGUO'hsa-Sirt1}

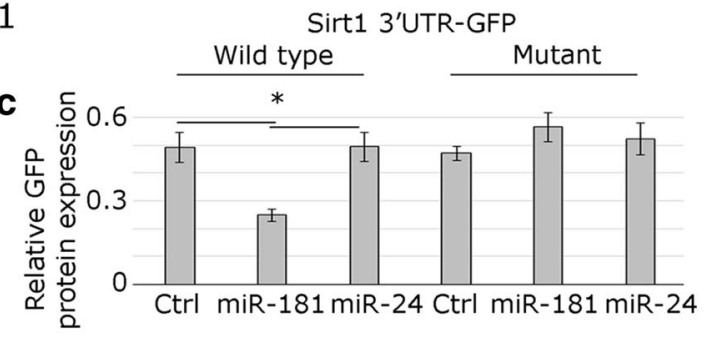

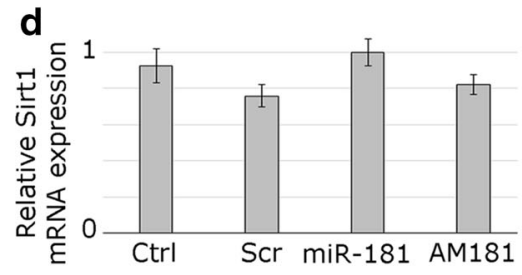

e

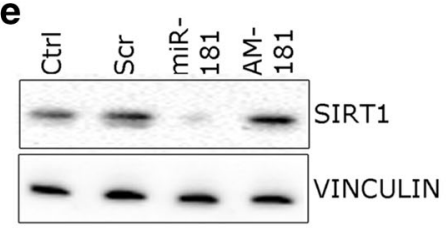

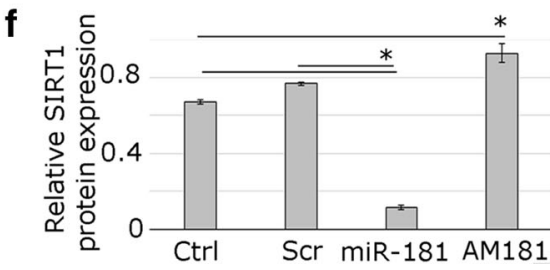

Fig. 4 miR-181a represses Sirtl expression in myotubes. a Alignment of putative miR-181a target site in the $3^{\prime}$ UTR of Sirt1 gene; human and mouse sequence are indicated; conserved miR-181a putative target site is indicated in red; complementary nucleotides are shown in orange, miR-181a seed sequence is shown in blue. b GFP-Sirt1 3'UTR sensor constructs containing conserved mouse wild type or mutated miR-181a target site were transfected into mouse myoblasts. Co-transfection with miR-181a mimic but not miR-24 mimic, led to downregulation of GFP protein expression compared to mock-transfected

These data suggest that changes in miR-181a and expression of its target gene Sirtl in skeletal muscle during ageing may indeed have functional consequences on muscle homeostasis, specifically myofibre hypertrophy and/or atrophy.

\section{Discussion}

The molecular mechanisms involved in sarcopenia development are not fully understood, however it is clear that transcriptomic, proteomic and epigenetic changes are involved. miRNAs can simultaneously modulate many signalling pathways and are therefore likely to be high-throughput regulators of pathophysiological changes associated with sarcopenia.

We have identified and validated miRNAs and mRNAs differentially expressed in skeletal muscle during ageing. In mouse muscle (TA), 30 miRNAs were differentially expressed during ageing (Table 1). Of these, 7 miRNAs were differentially expressed during ageing in the skeletal muscle of mice and control (Ctrl), as shown by representative western blot. Point mutations in the microRNA target site (mutant) rendered the sensor construct unresponsive. c Quantification of western blots is shown. d, e Endogenous SIRT1 protein but not mRNA expression is regulated by miR-181 in $\mathrm{C} 2 \mathrm{C} 12$ myotubes, as shown by representative Western blot or qPCR, respectively. f Quantification of Western blots is shown. Error bars show SEM; * $\mathrm{p}<0.05$ (compared with control or scrambled control as indicated); $\mathrm{n}=3$

humans based on data presented here and deposited in publically available databases (Drummond et al. 2011b; Rivas et al. 2014a). 5 microRNAs with a role in muscle biology: miR-26a (Dey et al. 2012), miR499 (van Rooij et al. 2009a), miR34b (Roberts et al. 2012), miR-30c (Ketley et al. 2013) and miR-181a (Naguibneva et al. 2006) were validated as differentially expressed during ageing in the skeletal muscle of mice (Fig. 3a). The differential expression of the miRNAs during ageing was validated in whole muscle rather than myofibres and therefore it is possible that age-related changes in muscle quality, such as adipocyte or immune cell infiltration, may be associated with changes in microRNA expression. Nevertheless, these data suggest that deregulation of microRNA expression during ageing may have functional consequences related to disrupted muscle homeostasis.

Previous studies have investigated changes in the expression of miRNAs in muscle during ageing (Drummond et al. 2011b; Hu et al. 2014b; Kim et al. 2014b), however it is noteworthy, that the overlap in 

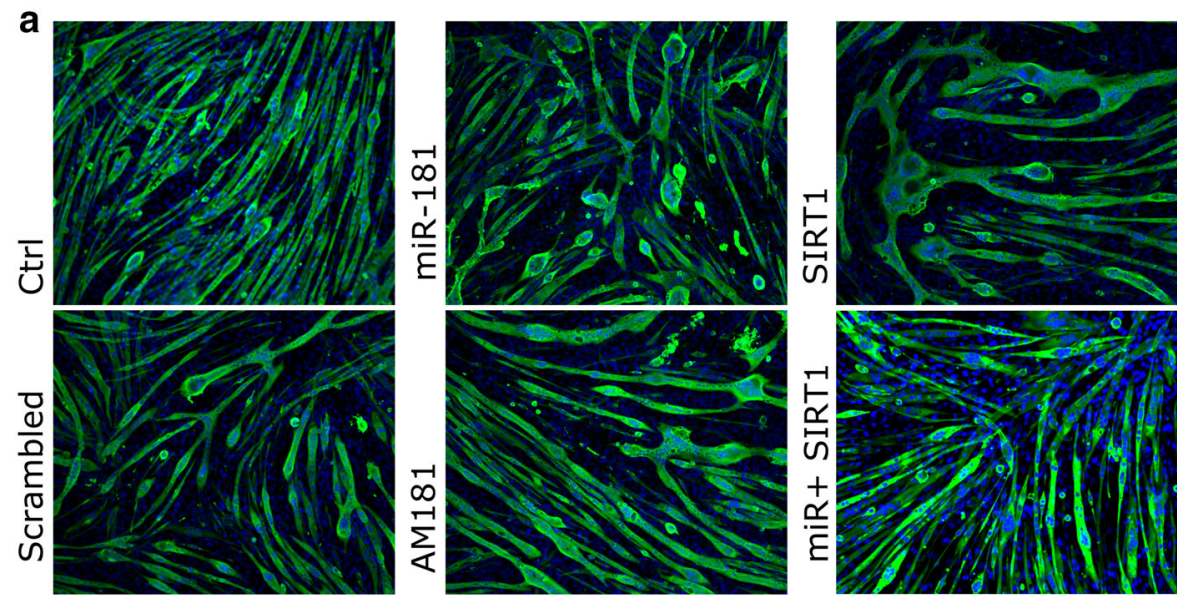

b

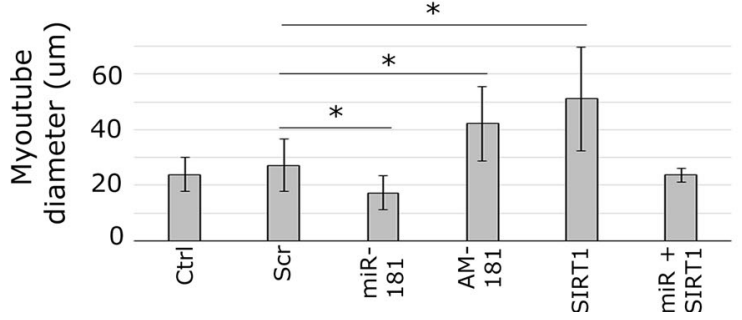

Fig. 5 miR-181 negatively regulates myotube size by targeting Sirt1. Expression of miR-181 and its target gene, Sirt1, was manipulated in $\mathrm{C} 2 \mathrm{C} 12$ myotubes; following transfections myotubes were stained for myosin heavy chain: MF20 - green;

deregulated miRNA expression in muscle of old animals between different studies is limited. This may be due to the use of different transcriptomic platforms or differential ageing processes in anatomically distinct muscle types. Standardising research methods used to globally profile changes in miRNA expression in different types of muscle and organisms would be an important step forward towards a better understanding of the role of microRNAs in the decline of muscle mass and function during ageing.

Using miRSystem and Ingenuity pathways, we modelled microRNA:target interactions affected by ageing. Our data indicates that deregulated miRNA:target interactions may be responsible for ageingrelated pathophysiological processes in skeletal muscle through regulation of ubiquitin-mediated proteolysis, ER stress and NFKB signalling, senescence and splicing factor expression, previously reported to be affected by ageing (Carvalho et al. 1996; Brack et al. 2007; Deldicque 2013; Holly et al. 2013; Drummond et al. 2014). Interestingly, Ubc, Ucp3 and IGF signalling pathways were also predicted to be a part
DAPI-blue. a MF20 immunostaining showing myotube size regulation by miR-181a and Sirt1. b Quantification of myotube diameter is shown $(\mu \mathrm{m})$. Error bars show SEM, $* \mathrm{p}<0.05$ (compared to control), $\mathrm{n}=4$

of the disrupted microRNA:target interactions during ageing (Fig. S2). As the anabolic action of insulin is reduced in muscle during ageing, and as muscle absorbs large amounts of circulating glucose, changes in IGF signalling in muscle during ageing could affect the levels of circulating glucose and therefore glucose present in other tissues, therefore affecting metabolism (Rasmussen et al. 2006).

Further analysis of the interactome of miRNAs differentially expressed during ageing in mouse and human muscle revealed that insulin, MAPK and TGF $\beta$ signalling, as well as ER stress, are likely to be regulated by these microRNAs; with miR-181a potentially playing a key role in age-associated changes in gene expression and signalling pathways in muscle. The analysis of miR-181a predicted targets supports this hypothesis, as miR-181a predicted target genes include genes/pathways with known role(s) in maintaining muscle homeostasis, for example Sirt1, Pten, and $p 38, T g f \beta, T n f \alpha$, NF-kB and insulin signalling pathways (Fig. S3). miR-181a has been previously shown to regulate muscle regeneration (Naguibneva 
et al. 2006) and downregulation of its expression may be also related to deterioration of satellite cell function and impaired regeneration of muscle during ageing.

miRNAs provide a potent and highly responsive mechanism enabling cells to react to changes in their environment by controlling cellular protein content through regulating the expression of multiple target genes. It is therefore important to characterise miRNA target gene(s) when establishing miRNA function. We have validated differential expression of miR-181a and mRNA and analysed protein expression of miR-181a predicted targets genes: Sirt1, Pten, Meox2, in the mouse TA muscle during ageing (Fig. 3). Sirtl is a member of the sirtuin family, a NAD+-dependent protein deacetylase known for its protective anti-ageing effects and also shown to promote muscle hypertrophy (Lee and Goldberg 2013; Sin et al. 2015). Meox2 controls muscle size and myofibre metabolism (Otto et al. 2010), whilst Pten inhibition improves muscle regeneration ( $\mathrm{Hu}$ et al. 2010). Among miR-181a predicted targets, SIRT1 protein levels were significantly changed in muscle during ageing (Fig. 3). We have validated Sirtl as a physiologically relevant direct miR-181a target in C2C12 myotubes (Fig. 4) and showed that miR-181 regulates myotube size through Sirt1, and potentially other target genes (Fig. 5). Since the expression of miR-181a is downregulated and Sirt1 expression is upregulated in muscle during ageing, and miR-181 negatively regulates myotube size, we suggest that age-related changes in miR-181a and its target gene(s) expression may act as a failing compensatory mechanism intended to preventing loss of muscle mass and potentially function. Previous data has shown that such compensatory mechanisms exist. For example, elevated levels of miR-206 in muscle of the amyotrophic lateral sclerosis (ALS) mouse model have been shown to compensate for the decreased neuromuscular interactions (Williams et al. 2009). Future experiments using in vivo model organism(s) will determine whether age-related changes in miR-181a expression may indeed act as a compensatory mechanism to maintain muscle mass and potentially function during ageing. It is noteworthy that $\mathrm{Hu}$ et al. (2014a, b) have shown that deregulation of miR-29 expression in muscle during ageing is associated with increased muscle senescence and therefore it is likely that changes in microRNA expression during ageing might act as compensatory or causative events, depending on the specific microRNA.
miRNAs present a potential to regulate a variety of pathophysiological conditions, such as sarcopenia. The ability to manipulate miRNA expression may offer therapeutic potential for ameliorating sarcopenia by modulating the rate of loss of muscle mass and function with age. However, it is necessary to establish the nature of changes in miRNA expression during ageing, as well as the factors triggering these changes. Furthermore, functional studies are needed to establish whether miRNAs initiate ageing-related changes in muscle homeostasis or fine-tune an already initiated process, and whether some microRNAs act in a compensatory manner to maintain muscle homeostasis in older individuals.

Acknowledgments The authors would like to thank Prof. Malcolm Jackson and Prof. Anne McArdle for their mentoring, Dr. George Sakellariou for his guidance with the techniques and discussions.

Author contributions KG-W, AS, LH performed the experiments, LT analysed the microarray data; EC-L provided advice to LT. All authors approved the final version of the manuscript.

Funding This work is supported by the Biotechnology and Biological Sciences Research Council (BBSRC; BB/L021668/ 1), the MRC and Arthritis Research UK as part of the MRC Arthritis Research UK Centre for Integrated Research into Musculoskeletal Ageing (CIMA) and the Wellcome Trust Institutional Strategic Support Fund (097826/Z/11/A).

\section{Compliance with ethical standards}

Conflict of interest The authors do not report any conflict of interest.

Open Access This article is distributed under the terms of the Creative Commons Attribution 4.0 International License (http:// creativecommons.org/licenses/by/4.0/), which permits unrestricted use, distribution, and reproduction in any medium, provided you give appropriate credit to the original author(s) and the source, provide a link to the Creative Commons license, and indicate if changes were made.

\section{References}

Bartel DP (2004) MicroRNAs: genomics, biogenesis, mechanism, and function. Cell 116:281-297

Brack AS, Conboy MJ, Roy S, Lee M, Kuo CJ, Keller C, Rando TA (2007) Increased Wnt signaling during aging alters muscle stem cell fate and increases fibrosis. Science 317:807-810 
Brown DM, Goljanek-Whysall K (2015) microRNAs: modulators of the underlying pathophysiology of sarcopenia? Ageing Res Rev 24:263-273

Brunet A, Sweeney LB, Sturgill JF, Chua KF, Greer PL, Lin Y, Tran H, Ross SE, Mostoslavsky R, Cohen HY, Hu LS, Cheng HL, Jedrychowski MP, Gygi SP, Sinclair DA, Alt FW, Greenberg ME (2004) Stress-dependent regulation of FOXO transcription factors by the SIRT1 deacetylase. Science 303:2011-2015

Carlson ME, Conboy IM (2007) Loss of stem cell regenerative capacity within aged niches. Aging Cell 6:371-382

Carlson ME, Suetta C, Conboy MJ, Aagaard P, Mackey A, Kjaer M, Conboy I (2009) Molecular aging and rejuvenation of human muscle stem cells. EMBO Mol Med 1:381-391

Carvalho BS, Irizarry RA (2010) A framework for oligonucleotide microarray preprocessing. Bioinformatics 26: 2363-2367

Carvalho CR, Brenelli SL, Silva AC, Nunes AL, Velloso LA, Saad MJ (1996) Effect of aging on insulin receptor, insulin receptor substrate-1, and phosphatidylinositol 3-kinase in liver and muscle of rats. Endocrinology 137:151-159

Cheung TH, Quach NL, Charville GW, Liu L, Park L, Edalati A, Yoo B, Hoang P, Rando TA (2012) Maintenance of muscle stem-cell quiescence by microRNA-489. Nature 482: 524-528

Crist CG, Montarras D, Pallafacchina G, Rocancourt D, Cumano A, Conway SJ, Buckingham M (2009) Muscle stem cell behavior is modified by microRNA-27 regulation of Pax3 expression. Proc Natl Acad Sci 106:13383-13387

Deldicque L (2013) Endoplasmic reticulum stress in human skeletal muscle: any contribution to sarcopenia? Front Physiol 4:236

Dey BK, Gagan J, Yan Z, Dutta A (2012) miR-26a is required for skeletal muscle differentiation and regeneration in mice. Genes Dev 26:2180-2191

Drummond MJ, McCarthy JJ, Sinha M, Spratt HM, Volpi E, Esser KA, Rasmussen BB (2011a) Aging and microRNA expression in human skeletal muscle: a microarray and bioinformatics analysis. Physiol Genomics 43:595-603

Drummond MJ, McCarthy JJ, Sinha M, Spratt HM, Volpi E, Esser KA, Rasmussen BB (2011b) Aging and microRNA expression in human skeletal muscle: a microarray and bioinformatics analysis. Physiol Genomics 43(10):595-603

Drummond MJ, Addison O, Brunker L, Hopkins PN, McClain DA, LaStayo PC, Marcus RL (2014) Downregulation of E3 ubiquitin ligases and mitophagy-related genes in skeletal muscle of physically inactive, frail older women: a crosssectional comparison. J Gerontol Ser A Biol Sci Med Sci 69:1040-1048

Friedman RC, Farh KK, Burge CB, Bartel DP (2009) Most mammalian mRNAs are conserved targets of microRNAs. Genome Res 19:92-105

Goljanek-Whysall K, Sweetman D, Abu-Elmagd M, Chapnik E, Dalmay T, Hornstein E, Munsterberg A (2011) MicroRNA regulation of the paired-box transcription factor Pax3 confers robustness to developmental timing of myogenesis. Proc Natl Acad Sci USA 108:11936-11941

Goljanek-Whysall K, Pais H, Rathjen T, Sweetman D, Dalmay T, Munsterberg A (2012) Regulation of multiple target genes by miR-1 and miR-206 is pivotal for $\mathrm{C} 2 \mathrm{C} 12$ myoblast differentiation. J Cell Sci 125:3590-3600
Goljanek-Whysall K, Mok GF, Fahad Alrefaei A, Kennerley N, Wheeler GN, Munsterberg A (2014) myomiR-dependent switching of BAF60 variant incorporation into Brg1 chromatin remodeling complexes during embryo myogenesis. Development 141:3378-3387

Holly AC, Melzer D, Pilling LC, Fellows AC, Tanaka T, Ferrucci L, Harries LW (2013) Changes in splicing factor expression are associated with advancing age in man. Mech Ageing Dev 134:356-366

Hu Z, Wang H, Lee IH, Modi S, Wang X, Du J, Mitch WE (2010) PTEN inhibition improves muscle regeneration in mice fed a high-fat diet. Diabetes 59:1312-1320

Hu Z, Klein JD, Mitch WE, Zhang L, Martinez I, Wang XH (2014a) MicroRNA-29 induces cellular senescence in aging muscle through multiple signaling pathways. Aging (Albany NY) 6:160

Hu Z, Klein JD, Mitch WE, Zhang L, Martinez I, Wang XH (2014b) MicroRNA-29 induces cellular senescence in aging muscle through multiple signaling pathways. Aging 6:160-175

Jackson MJ, McArdle A (2011) Age-related changes in skeletal muscle reactive oxygen species generation and adaptive responses to reactive oxygen species. J Physiol 589: 2139-2145

Ketley A, Warren A, Holmes E, Gering M, Aboobaker AA, Brook JD (2013) The miR-30 microRNA family targets smoothened to regulate hedgehog signalling in zebrafish early muscle development. PLoS ONE 8:e65170

Kim JY, Park Y-K, Lee K-P, Lee S-M, Kang T-W, Kim H-J, Dho SH, Kim S-Y, Kwon K-S (2014a) Genome-wide profiling of the microRNA-mRNA regulatory network in skeletal muscle with aging. Aging (Albany NY) 6:524

Kim JY, Park YK, Lee KP, Lee SM, Kang TW, Kim HJ, Dho SH, Kim SY, Kwon KS (2014b) Genome-wide profiling of the microRNA-mRNA regulatory network in skeletal muscle with aging. Aging 6:524-544

Lee D, Goldberg AL (2013) SIRT1 protein, by blocking the activities of transcription factors FoxO1 and FoxO3, inhibits muscle atrophy and promotes muscle growth. J Biol Chem 288:30515-30526

McCarthy JJ, Esser KA (2007) MicroRNA-1 and microRNA133a expression are decreased during skeletal muscle hypertrophy. J Appl Physiol 102:306-313

McDonagh B, Sakellariou GK, Jackson MJ (2014) Application of redox proteomics to skeletal muscle aging and exercise. Biochem Soc Trans 42:965-970

Mercken EM, Majounie E, Ding J, Guo R, Kim J, Bernier M, Mattison J, Cookson MR, Gorospe M, de Cabo R (2013) Age-associated miRNA Alterations in Skeletal Muscle from Rhesus Monkeys reversed by caloric restriction. Aging (Albany NY) 5:692

Naguibneva I, Ameyar-Zazoua M, Polesskaya A, Ait-Si-Ali S, Groisman R, Souidi M, Cuvellier S, Harel-Bellan A (2006) The microRNA miR-181 targets the homeobox protein Hox-A11 during mammalian myoblast differentiation. Nat Cell Biol 8:278-284

Otto A, Macharia R, Matsakas A, Valasek P, Mankoo BS, Patel K (2010) A hypoplastic model of skeletal muscle development displaying reduced foetal myoblast cell numbers, increased oxidative myofibres and improved specific tension capacity. Dev Biol 343:51-62 
Rasmussen BB, Fujita S, Wolfe RR, Mittendorfer B, Roy M, Rowe VL, Volpi E (2006) Insulin resistance of muscle protein metabolism in aging. FASEB J 20:768-769

Ritchie ME, Diyagama D, Neilson J, van Laar R, Dobrovic A, Holloway A, Smyth GK (2006) Empirical array quality weights in the analysis of microarray data. BMC Bioinform $7: 261$

Ritchie ME, Phipson B, Wu D, Hu Y, Law CW, Shi W, Smyth GK (2015) limma powers differential expression analyses for RNA-sequencing and microarray studies. Nucleic Acids Res 43:e47

Rivas DA, Lessard SJ, Rice NP, Lustgarten MS, So K, Goodyear LJ, Parnell LD, Fielding RA (2014a) Diminished skeletal muscle microRNA expression with aging is associated with attenuated muscle plasticity and inhibition of IGF-1 signaling. FASEB J 28:4133-4147

Rivas DA, Lessard SJ, Rice NP, Lustgarten MS, So K, Goodyear LJ, Parnell LD, Fielding RA (2014b) Diminished skeletal muscle microRNA expression with aging is associated with attenuated muscle plasticity and inhibition of IGF-1 signaling. FASEB J 28:4133-4147

Roberts TC, Blomberg KE, McClorey G, El Andaloussi S, Godfrey C, Betts C, Coursindel T, Gait MJ, Smith CI, Wood MJ (2012) Expression analysis in multiple muscle groups and serum reveals complexity in the microRNA transcriptome of the $\mathrm{mdx}$ mouse with implications for therapy. Mol Therapy 1:e39

Sifakis EG, Valavanis I, Papadodima O, Chatziioannou AA (2013). Identifying gender independent biomarkers responsible for human muscle aging using microarray data. In Bioinformatics and Bioengineering (BIBE), 2013 IEEE 13th International Conference oned^eds): IEEE, pp 1-5

Sin TK, Yung BY, Siu PM (2015) Modulation of SIRT1-Foxo1 signaling axis by resveratrol: implications in skeletal muscle aging and insulin resistance. Cell Physiol Biochem 35:541-552
Smyth GK (2004). Linear models and empirical bayes methods for assessing differential expression in microarray experiments. Stat Appl Genet Mol Biol, 3: Article3

Smyth GK, Michaud J, Scott HS (2005) Use of within-array replicate spots for assessing differential expression in microarray experiments. Bioinformatics 21:2067-2075

Soares RJ, Cagnin S, Chemello F, Silvestrin M, Musaro A, De Pitta C, Lanfranchi G, Sandri M (2014) Involvement of microRNAs in the regulation of muscle wasting during catabolic conditions. J Biol Chem 289:21909-21925

Soriano-Arroquia A, McCormick R, Molloy AP, McArdle A, Goljanek-Whysall K (2016). Age-related changes in miR143-3p:Igfbp5 interactions affect muscle regeneration. Aging cell

van Rooij E, Quiat D, Johnson BA, Sutherland LB, Qi X, Richardson JA, Kelm RJ Jr, Olson EN (2009a) A family of microRNAs encoded by myosin genes governs myosin expression and muscle performance. Dev Cell 17:662-673

Van Rooij E, Quiat D, Johnson BA, Sutherland LB, Qi X, Richardson JA, Kelm RJ, Olson EN (2009b) A family of microRNAs encoded by myosin genes governs myosin expression and muscle performance. Dev Cell 17:662-673

Welle S, Brooks AI, Delehanty JM, Needler N, Thornton CA (2003) Gene expression profile of aging in human muscle. Physiol Genomics 14:149-159

Williams AH, Valdez G, Moresi V, Qi X, McAnally J, Elliott JL, Bassel-Duby R, Sanes JR, Olson EN (2009) MicroRNA206 delays ALS progression and promotes regeneration of neuromuscular synapses in mice. Science 326:1549-1554

Zacharewicz E, Della Gatta P, Reynolds J, Garnham A, Crowley T, Russell AP, Lamon S (2014) Identification of microRNAs linked to regulators of muscle protein synthesis and regeneration in young and old skeletal muscle. PLoS ONE 9:e114009 